ORIENTAL JOURNAL OF
ISSN: 0974-6471
March 2017,
$\begin{gathered}\text { Col. 10, No. (1): } \\ \text { Pgs. 238-248 }\end{gathered}$

\title{
Age Estimation using OLPP Features
}

\author{
M S VAISHNAVI* and A VIJAYALAKSHMI \\ Department of Computer Science, Christ University, Hosur road, Bangalore, India. \\ ${ }^{*}$ Corresponding author E-mail: vaishnavi.ms.24@gmail.com \\ http://dx.doi.org/10.13005/ojcst/10.01.33
}

(Received: March 17, 2017; Accepted: March 18, 2017)

\begin{abstract}
Aging face recognition poses as a key difficulty in facial recognition. It refers to identification of a person face over varied ages. It includes issues like age estimation, progression and verification. Non-availability of facial aging databases make it harder for any system to achieve good accuracy as there are no good training sets available. Age estimation when done correctly has a varied number of real life applications like age detailed vending machines, age specific access control and finding missing children. This paper implements age estimation using Park Aging Mind laboratory - Face database that contains meta data and 293 unique images of 293 individuals. Ages range from 19 to 45 with a median age of 32 . Race is classified into two categories : African-American and Caucasian giving an accuracy of $98 \%$. Sobel edge detection and Orthogonal locality preservation projection were used as the dominant features for the training and testing of age estimation. A Multi-stage binary classification using support vector machine was used to classify images into an age group thereafter predicting an individual's age. The effectiveness of this method can be increased by using a large data set with a wider age range.
\end{abstract}

Keywords: Face recognition, Orthogonal Locality Preservation Projections, Age Estimation, Multi-Stage Support Vector Machine.

\section{INTRODUCTION}

Security is a property which measures the degree of resisting some harmful attack on any vulnerable entity. The entity can represent anything that exists in the nature. It can represent some human being, object, company, country, etc. All these are associated with security in order to achieve their goals and objectives. Any entity can be vulnerable to any kind of attack or harm. It is the security of that system which makes it strong enough to defend against those harmful attacks and tackle the situation accordingly. Every system essentially needs some security mechanism. The main reason for the systems to need security is the rapid development in the field of information processing. Data and information are continuously increasing day by day. This makes it essential to provide some security mechanisms in order to protect the data. It is unacceptable when situations occur where information is lost or modified. Some entities which perform in real-time mode are totally dependent on the integrity of the information that is being processed. Normally data security is compromised during the process of transmission. Any attacker can meddle with the information that is 
being transmitted across a certain medium. Attacks can occur even when the information is not being transmitted. In order to avoid all these serious circumstances, we essentially need security to any system that we define.

There are many ways wherein we can provide security. Some of them can be listed as:

- Using Personal Identification Number
- $\quad$ UIN)
- $\quad$ Using passwords
- $\quad$ Using location based authentication
Usiacial recognition, etc.

One of the most useful ways to provide security is to use the facial recognition technique. In this technique, a picture of a person is taken and some pre-processing is done. It will then be checked if that particular picture is present in the database or not. If it is not, then it will be added to it and some parameters will be set accordingly. The picture present in the database could be an old picture of the same person. But we cannot refuse authorizing him just because the face looks similar and the same. The currently obtained facial will be processed to extract some values which will be compared to the values of the old picture and based on this result the person will either be authenticated or some suitable message will be displayed on error.

Biometric security involves the actual human interaction with a system to authenticate the user. This proves to be an efficient system as it uses the characteristics of human body and parts to provide security. Every human body is unique and can never be the same as other. There are many ways through which we can achieve this. Most common among them are Fingerprint scanner and facial recognition system. Every individual's fingerprint is not same and can never be the same for anyone. The person will be authenticated if his fingerprint matches with the fingerprint present in his database entry. A more efficient way of achieving biometric security is to use the facial recognition system. This is more preferred as it has no direct contact of the human body with the machine or the system. This doesn't require any direct interaction with the person. This can also be used in the crime detection purposes. Once a person's face is recorded in the database, there is nothing that can prove it wrong when it is being cross-checked. This can be implemented as a functionally independent component of a system.

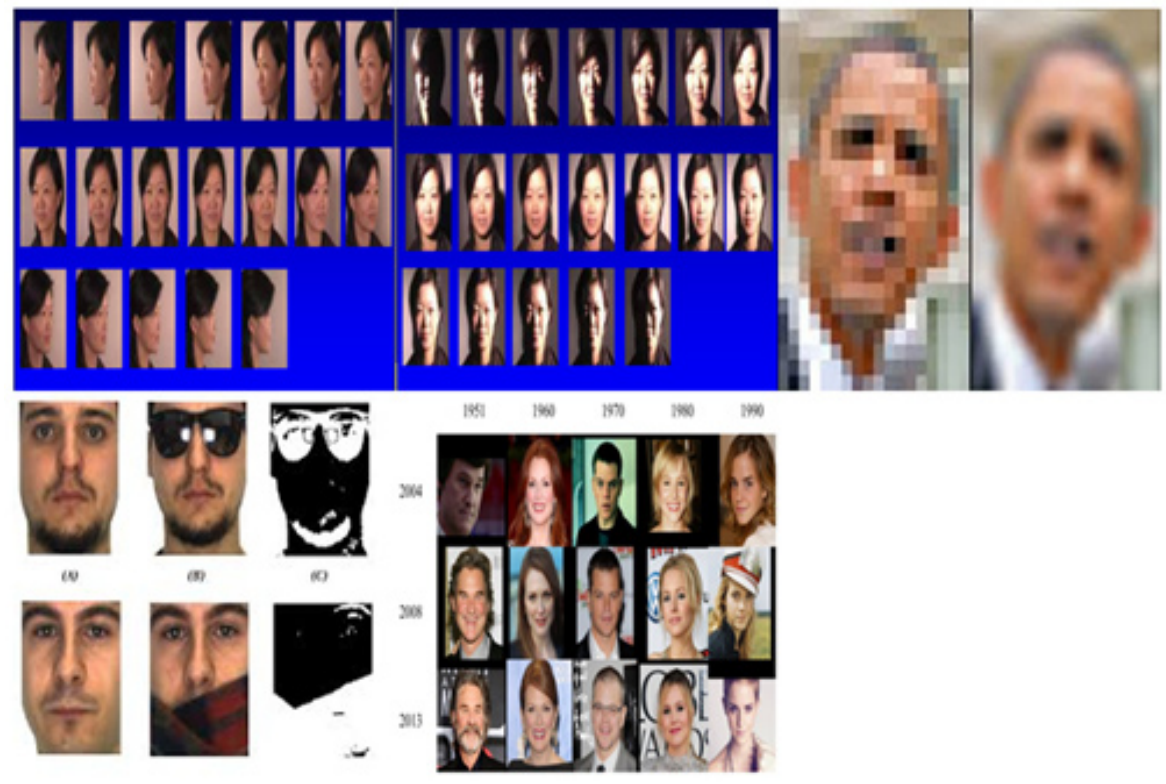

Fig. 1.1: Challenges faced in facial recognition With respect to Pose, Illumination, Resolution, Occlusion and Aging 
Facial recognition has several uses in daily actions, it allows identity verification of individuals in banking transactions, online payments, control access to high security facilities etc. However lately facial recognition is widely used to uniquely authenticate an individual as this technology doesn't require a contact between the sensor and the individual, which enables remote authentication and authorisation. Some of the advantages of facial recognition are that it can be operated without the cooperation of actual human beings. These devices when properly installed can be used in companies, malls, airports and other similar vulnerable areas. This can also be used in public places without people getting to know about it. This is among the key methods used in mass identification.

Face recognition comes with its own set of challenges like variation of Pose, Illumination, Occlusion, low resolution and aging.

The drawbacks in this system which does need some special attention. It might not work in some unusual and strange circumstances. The simple example being, recognizing a face from a different angle. This will pose a problem if we try to compare two faces where one of them is deviated in an angle which is more than the one that can be used. One more disadvantage is that the system becomes less effective if a complex facial recognition system is used. Facial expressions should be similar while comparing. All these make it a not-so-perfect technique to be used while providing security.

Aging is an unescapable process. Aging causes major differences in the outlook of human faces over a period of time. Some features of aging are controllable whilst others are not. The uncontrollable features are commonly based on hereditary elements and the controllable features are a result of lifestyle choices like nutrition, smoking, stress, genetics etc. Aging face recognition and age estimation has various beneficial real-world applications like identifying mugshots and criminals based on photographs or finding missing people mainly children.

Aging is an uncontrollable and un escapable process. It still remains the toughest drawback of facial recognition left to overcome. Hereditary and lifestyle choices make it harder for any system to estimate a person's age. The system must identify or estimate the age of a person for validation and progression. Once the age is correctly estimated it can further be used to perform facial recognition, age progression and age simulation for crime related or verification related purposes like guessing the current age of a missing person. This system can also be used to validate the age progression and age simulation projects available in the market like My Web Face, In20years, Face Transformer...

The appearance of a human face is affected significantly by aging. Facial aging effects vary in the childhood age to that of the adult age. Aging effects include bone movement, bone growth, skin related changes like wrinkles and reduction of muscle strength. Usually craniological growth of the facial bones takes place during childhood which determines the shape and the structure of the face whereas during adult ages textural changes like skin deformations occur. However, researchers who carried out work in studying the process of age estimation by humans (Rhodes 2009) conclude that humans are not so accurate in age estimation hence the possibility of developing automatic facial age estimation methods were proposed.

In automatic facial age estimation the goal is to use algorithms to determine a person's age. It includes face detection, feature extraction, feature vector formulation and classification. The output of the classification can either be an estimated age, age group or even a binary result indicating if the age belongs within an age range. Age classification is the preferred output as it only a rough estimate of a person's age is required. Thus range considered also becomes an important factor as facial features vary over different age groups. The commonly used error metric for age estimation problems are Mean Absolute Error (MAE) and Cumulative Score (CS). MAE provides the actual and the estimated faces in a test set whereas CS that offers a percentage where the estimation error is less than a threshold. Both methods can be used to for performance evaluation of the system.

Age estimation system gives the 
chronological age (actual age), Appearance age and the difference between them. Some real life applications of age estimation are:

- Age-specific access control

- $\quad$ Children protection

- Security and Surveillance in Law enforcement

- Multi-cue identification as face/fingerprint/ iris + age

- Internet safety for minors

- $\quad$ Cigarette vending machines

- $\quad$ Age specific shopping $\mathrm{HCl}$

- Data mining and organisation

\section{Previous Work}

Face recognition contains face identification, verification and matching. Face identification and verification (Richa Singh et al, 2007) is implemented by suggesting an algorithm which lists the gallery and probes face image in Polar Coordinate domain. It lessens the facial feature changes which occur due to aging. It takes the face image of the current and former and generates a new image based on the phase congruency. It uses local feature extraction and resolution to determine the assorted form of the face image. It attains an enhancement in Facial recognition and authentication by about $25 \%$.

There are four main challenges in facial recognition. An article study (A.K.Jain et al, 2012) based on four challenges in facial recognition; pose, illumination, expression and aging studies the forensic and non-forensic procedures used in Law Enforcement. It tries to use ranked search to recognise the identical images such that after the outcome of the ranked image, a manual $1: \mathrm{N}$ search will easily help distinguish the right face image. It concludes by saying that filtering is challenging but usually gender and age filters out the images quite easily. A study report (A.K.Jain et al, 2011) on aging invariant Face detection and recognition through identification of facial marks and features vs the recognition through sketch is studied and results have been provided. Face recognition in video as well as 3D modelling of face is studied and analysed indicating that automatic face recognition is better.

Due to a great rise in law enforcement, security and human-computer interaction, the field of age estimation using facial images has a growing interest in these recent years. This might be also affected by factors such as intrinsic (genetic) and extrinsic factors (lifestyle). Due to these factors people of different ages might have the similar facial images. The main theory is to develop a hierarchical approach for automatic age estimation. In a paper (H.Han et al, 2015) proposes to carry out pre-processing, component localization, feature extraction, hierarchical age estimation, fusion and age estimation by using the crowd-sourced data. This uses the FG-NET, MORPH Album2 and PCSO databases. The performance was seen the best on the Morph Album2 database.

Although a lot of research work has been implemented in overcoming facial recognition challenges, aging still poses a major challenge in facial recognition.

Whereas a new method $(\mathrm{H}$. Ling et al, 2007) uses a gradient orientation pyramid with a two class problematic resolving through support vector machine (SVM) to study facial recognition within a real passport photo verification mission. The SVM acts as a classifier which produces a strong and discriminative representation. It has been established that although this method outdoes Bayesian Method of face recognition, the aging, illumination and expression features adds trouble to the recognition task. Where as in another paper (Z. Li et al,2016) use a local pattern selection (LPS) model to reduce intra-user dissimilarity at a microstructure level using feature encoder. The pixels are then clustered into partitions based on multiple scaling and dense sampling techniques. Then they use a scalable high-level visual information refinement structure to reserve only the most vital information for face recognition. LPS on MORPH provides accuracy of $92.11 \%$ whereas LPS + HOG descriptor provides accuracy of $94.20 \%$ and LPS + HFA provides accuracy of $94.87 \%$.

Face Recognition as People Age is still a major challenge faced by the industry. $A$ paper $(H$. Yang, 2016) attains aging simulation using Hidden Factor Analysis (HFA) on age variant features. This method separates the age-specific facial signs that change gradually over time. It then operates 
on age, by changing it to a target age group using sparse reconstruction, to add aging effects on the face texture and then enhances it by facial shape transformation. The aging and rejuvenating results attained on MORPH, FG-NET, IRIP show that the generated aging rendering are consistent and substantial.

Whereas in another method (J. Qiu et al ,2016) presents a new aggregation based deep network to extract aging features from facial images on age variant features to achieve age detection and prediction. They employ a regionspecific convolutional neural network (CNN) at lower layers that are hierarchically aggregated into consecutive higher layers. Experimental results of age prediction on the MORPH-II databases prove that this outperforms other state-of-the-art systems with a mean error of 3.41 for age detection and is relatively reliable and robust across race at 4.72 and gender at 4.43 .

In general, a paper (B. Esme et al, 2016) focuses on explaining that there are Age-Invariant features present on the face which can be mined through image processing and can be used to identify the face. It analyses generative and a non-generative approach which uses a simulated modelling of the human to assume the face features after aging.

Linear data transformation like Principle Component Analysis (PCA), Linear Discriminant Analysis (LDA), Local Binary Patterns (LBP), Locality Preservation Projections (LPP) help achieve good results for age simulation, progression and estimation compared to non-linear transformations.

Face detection and tracking, gender detection and age classification and in-cloud data statistics model are the 5 important and also the consecutive stages of real-time audience measurement system. Since this uses the machine learning methods, it is a bit challenging to do it. A paper $(\mathrm{H}$. Han et al, 2015) proposes a new algorithm which consists of two stages, i.e., adaptive feature extraction based on local binary patterns (LBP) and support vector machine (SVM) classification. This uses its RUS-FD (private), FG-NET and MORPH databases. Processing is done by using crowds ourcing too. However, age estimation from the unrestrained faces, still remains a difficult problem for such algorithms.

In 'Investigating Age Invariant Face Recognition Based on Periocular Biometrics' (F. Juefei-xu et al,2011), they use a Walsh-Hadamard transform encoded local binary patterns (WLBP) on only the pre-processed periocular region. They perform the same operations on various images across the age of the same individual and using unsupervised discriminant projection (UDP) to build subspaces on WLBP featured periocular images, they achieved $100 \%$ rank- 1 face identification rate and $98 \%$ face verification rate at $0.1 \%$ false accept rate on the entire FG-NET database.

An algorithm (B. F. Klare et al, 2011) suggests a method to match sketches to faces in a database through forensic algorithm. To identify forensic sketches, they use a structure called local feature-based discriminant analysis (LFDA). LFDA represents both sketches and photos using SIFT feature descriptors and multiscale local binary patterns (MLBP) and minimum distance matching. The paper concludes with a sizeable advance using a collection of 159 forensic sketches matched against a gallery with 10,159 mug shot images using FaceVACS database. However (S. Wang et al, 2015) uses a discriminative model where they denote each face by designing a densely sampled local feature description scheme using scale invariant feature transform (SIFT) and multiscale local binary patterns (MLBP) as the local descriptors. A multi-feature discriminant analysis (MFDA) was developed to process these two local feature spaces in a unified framework. Their method on evaluation has been proven to escalate the face matching accuracy to almost $83.6 \%$.

Using techniques like these age estimation is also made easier. It helps to identify and verify individuals. In 'Relative Attribute SVM + Learning for Age Estimation' (S. Wang et al, 2015)they suggest a raSVM+ (relative attribute SVM+) algorithm to differentiate the comparative age group. This approach utilized relative attributes as private data for raSVM+ learning, which improved the precision of age estimation by adjusting age determination errors during model training, and thus predicts 
a generalizable answer in the training datasets. raSVM+ is a hopeful growth that advances age estimation, with the mean absolute error of 4.07 on FG-NET and $5.05+/-0.11$ on MORPH.

Dimensionality reductions are a form of problems faced during information processing. Some linear projective maps such as Locality Preserving Projections (LPP) should be seen as an alternative to the Principal Component Analysis (PCA), where the neighbourhood data set is optimally preserved after solving a variation problem. In an ambient space, the LPP can be obtained by figuring out the optimal linear approximations to the Eigen functions of the Laplace Beltrami operator on the manifold, which is formed when high dimensional data lies on the low dimensional data. The linear approximation of the nonlinear Laplacian Eigen map is the concept of LPP. In 'Locality Preserving Projections' algorithm ( Xiaofei He, 2014), the adjacency graph will be constructed at the first, then weights are chosen and Eigen maps are generated. These maps are simple, linear and defined everywhere. It is capable of discovering the non-linear structure of data manifold even though this is a linear algorithm. But LPP has a limitation where it cannot solve the recognition problem. To overcome this problem, 'Face recognition using discriminant Locality Preserving Projections' paper (W. Yu et al, 2006) proposes a new method called Discriminant Locality Preserving Projections (DLPP) which benefits in two aspects where subspace is found to discriminate various facial classes and also reducing the noise. DLPP is better than LPP in terms of recognition accuracy. But in some other cases it is as efficient as LPP.

\section{MATERIALS}

\section{Data Collection}

Availability of facial databases forms the biggest obstacle in facial recognition. There are several databases for the study of the pose, illumination, resolution and expression. Procuring these databases is easy as they were created by taking photos of subjects under controlled conditions by changing one variable at a time. Unfortunately the same cannot be performed for facial ageing database as we would have to gather the same subject's photos over a number of years.
Apart from the images available for other purposes there are a number of images available on the internet. Nonetheless it is highly unreliable as it is difficult to find the age of the subject in the image. Even the application of makeup and plastic surgery poses as a problem as it masks the facial characteristics that help determine facial age.

The age estimation algorithm was developed and tested using the non-commercial version of the Park Aging Mind laboratory - Face database (Minear, M. \& Park, D.C. ,2004). This was a controlled collection of images (i.e., the images were not collected in real-world conditions containing noise and various forms of image distortion or obstruction). The dataset contained metadata in the form of age, gender, race, color, expression in .bmp file format. The collection included the total of 290 unique images of 290 individuals. Ages range from 19 to 45 with a median age of 32 .

Table I and Table II show the distribution of images used in this study by gender and ancestry and by age respectively. It can be observed that the gender distribution was balanced. Similarly there was an uneven ancestry distribution with Caucasian origin represented by clear majority of pictures. Looking at age distribution the range 19-45 was quite unevenly distributed with a clear majority of the age range 18-29, however the ranges $<19$ and $45+$ were unavailable.

Table 1: Data Distribution by Gender and Ethnicity

\begin{tabular}{lccc}
\hline & $\begin{array}{c}\text { African- } \\
\text { American }\end{array}$ & Caucasian & Total \\
\hline MALE & 56 & 89 & 145 \\
FEMALE & 39 & 109 & 148 \\
TOTAL & 95 & 198 & 293 \\
\hline \multicolumn{4}{c}{ Table 2: Data Distribution by } \\
\hline \multicolumn{5}{c}{ Age } \\
\hline MALE & $\mathbf{1 8 - 2 9}$ & $\mathbf{3 0 - 4 9}$ & TOTAL \\
FEMALE & 116 & 29 & 145 \\
TOTAL & 106 & 42 & 148 \\
\hline
\end{tabular}




\section{METHODS}

\section{Pre-Processing}

The raw facial images were pre-processed. The pre-processing stage included face and eye position detection, angle normalization, image resizing. These procedures were implemented using the Viola-Jones algorithm cascade object detectors for facial and facial feature recognition. To aid the edge detection algorithm, histogram equalisation and image sharpening was applied on the facial image to enhance the edges in the image. The final step of image pre-processing included the detection of person's ethnicity. The ethnicity detection algorithm iteratively scanned all facial regions using a small spatial window and calculated average values of the pixel color intensities within each window. The race was then determined by comparing these values with an arbitrary threshold determined experimentally.

\section{Feature Extraction}

\section{Sobel Features}

The sobel filter, sometimes called as the sobel operator is used in computer vision and image processing for mainly edge detection to create highlighting edges. It is a discrete differentiation operator, computing an approximation of the gradient of the image intensity function. The result of the Sobel-Feldman operator is either the corresponding gradient vector or the norm of this vector. It is inexpensive in terms of computations as it is based on convolution of the image with a small, separable, and integer-valued filter in the horizontal and vertical direction.

The sobel operator uses two $3 \times 3$ kernels - one for horizontal changes, and one for vertical which are convolved with the original image to calculate approximations of the derivatives. If we define $A$ as the source image, and Gx and Gy are two images which at each point contain the horizontal and vertical derivative approximations respectively, the computations are as follows ${ }^{17}$ :

$$
\begin{aligned}
G_{z} & =\left[\begin{array}{rrr}
-1 & 0 & +1 \\
-2 & 0 & +2 \\
-1 & 0 & +1
\end{array}\right] * A \\
G_{y} & =\left[\begin{array}{rrr}
-1 & -2 & -1 \\
0 & 0 & 0 \\
+1 & +2 & +1
\end{array}\right] * A
\end{aligned}
$$

Where here denotes the 2-dimensional signal processing convolution operation.

Since the Sobel kernels can be decomposed as the products of an averaging and

The algorithmic procedure for Sobel edge detection method is formally stated below:

\section{Input: A Sample Image}

Output: Detected Edges

Step 1: Accept the input image

Step 2: Apply mask Gy Gx, to the input image

Step 3: Apply Sobel edge detection algorithm and the gradient

Step 4: Masks manipulation of Gy Gx, separately on the input image

Step 5: Results combined to find the absolute magnitude of the gradient

$$
\mathrm{G}=\sqrt{G_{x}^{2}+G_{y}^{2}}
$$

Step 6: The absolute magnitude is the output edges

Fig. 5.1: Algorithm for sobel edge detection method(O. R. Vincent et al, 2009) 
a differentiation kernel, they compute the gradient with smoothing. For example, can be written as

$$
\left[\begin{array}{lll}
-1 & 0 & +1 \\
-2 & 0 & +2 \\
-1 & 0 & +1
\end{array}\right]=\left[\begin{array}{l}
1 \\
2 \\
1
\end{array}\right]\left[\begin{array}{lll}
-1 & 0 & +1
\end{array}\right]
$$

The $x$-coordinate is defined here as increasing in the "right"-direction, and the $y$-coordinate is defined as increasing in the "down"direction. At each point in the image, the resulting gradient approximations can be combined to give the gradient magnitude, using:

$$
\mathrm{G}=\sqrt{G_{z}{ }^{2}+G_{y}{ }^{2}}
$$

Using this information, we can also calculate the gradient's direction:

$$
\theta=\operatorname{atan} \frac{G_{y}}{G_{z}}
$$

Where, for example, $\Theta$ is 0 for a vertical edge which is lighter on the right side.

\section{OLPP Features}

The Sobel features are subjected to orthogonal locality preservation projection (OLPP) algorithm. Locality Preserving Projections (LPP) are linear projective maps that arise by solving a vibrational problem that optimally preserves the neighbourhood structure of the data set. LPP should be seen as an alternative to Principal Component Analysis (PCA) - a classical linear technique that projects the data along the directions of maximal variance. LPP shares many of the data representation properties of nonlinear techniques such as Laplacian Eigen maps or Locally Linear Embedding. Yet LPP is linear and more crucially is defined everywhere in ambient space rather than just on the training data points. LPP may be conducted in the original space or in the reproducing kernel Hilbert space into which data points are mapped. In simple words LPP keeps local structure of data intact. I.e. they keep data points near to a data point as nearly as possible.

The LPP algorithm is as follows:

Locality Preserving Projection (LPP) is a linear approximation of the nonlinear Laplacian Eigen map (A. K. Jain et al, 2012)

The OLPP has the orthogonally property. It builds an adjacency map which best represents the geometry of the low dimension facial manifold and class attributes between the sample points. In this way, the OLPP preserves the locality characteristics of images compared to that of LPP which is nonorthogonal, which prohibits data reconstruct after the dimension reduction is performed.

\section{Example Results after applying Sobel edge detection}
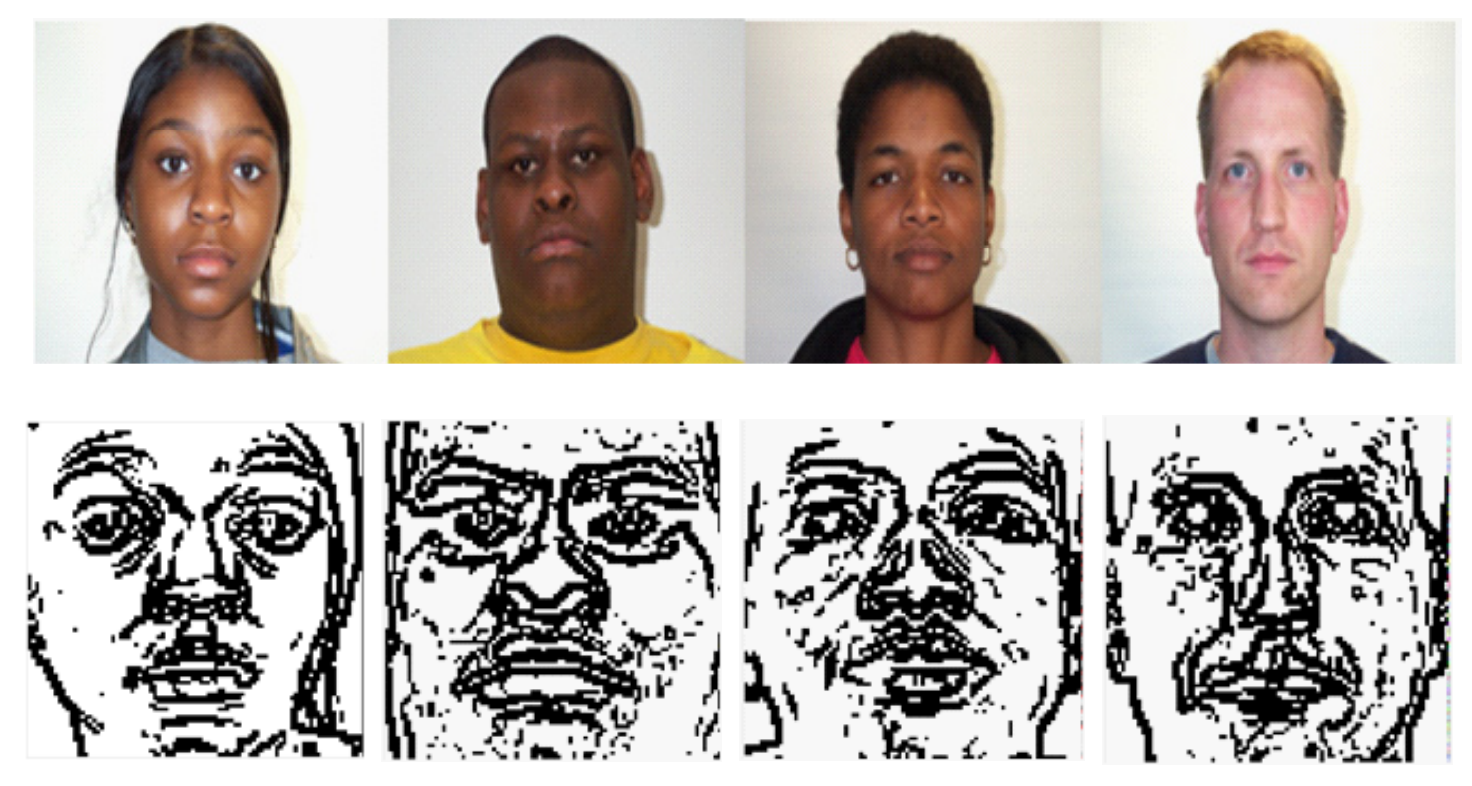
The algorithmic procedure is formally stated below:

1. Constructing the adjacency graph: Let $G$ denote a graph with $m$ nodes. We put an edge between nodes $i$ and $j$ if $\mathrm{x}$ and $\mathrm{x} j$ are "cloge". There are two variations:

(a) $\varepsilon$-neighborhoods. [parameter $\varepsilon \in \mathrm{R}$ ] Nodes $i$ and $j$ are connected by an edge if $\left\|x_{i}-x_{i}\right\|^{2}<\varepsilon$ where the norm is the usual Euclidean norm in $\mathbf{R}^{\prime}$.

(b) $k$ nearest neighbours. [parameter $k \in \mathrm{N}$ ] Nodes $i$ and $j$ are connected by an edge if $i$ is among $k$ nearest neighbours of $j$ or $j$ is among $k$ nearest neighbours of $i$. Note: The method of constructing an adjacency graph outlined above is correct if the data actually lie on a low dimensional manifold. In general, however, one might take a more utilitarian perspective and construct an adjacency graph based on any principle (for example, perceptual similarity for natural signals, hyperlink structures for web documents, etc.). Once such an adjacency graph is obtained, LPP will try to optimally preserve it in choosing projections.

2. Choosing the weights: Here, as well, we have two variations for weighting the edges. $W$ is a sparse symmetric $m \times m$ matrix with $W_{i 1}$ having the weight of the edge joining vertices $i$ and $j$, and 0 if there is no such edge.

(a) Heat kemel. [parameter $t \in \mathbf{R}$ ]. If nodes $i$ and $j$ are connected, put

$$
W_{i j}=e^{-\frac{\left|x_{i}-x_{j}\right|^{2}}{t}}
$$

The juatification for this choice of weights can be traced back to [2].

(b) Simple-minded. [No parameter]. $W_{i}=1$ if and only if vertices $i$ and $j$ are connected by an edge.

3. Eigenmaps: Compute the eigenvectors and eigenvalues for the generalized eigenvector problem:

$$
X Z X^{4} \mathrm{a}=\lambda X D X^{r} \mathrm{a}
$$

where $D$ is a diagonal matrix whose entries are column ( or row, since $W$ is symmetric) sums of $W, D_{x}=\sum j W_{3}$.

$L=D-W$ is the Laplacian matrix. The $i^{t}$ column of matrix $\mathrm{X}$ is $\mathbf{x}$. Let the column vectors $\mathbf{a}_{1}, \cdots, \mathbf{a}_{-1}$ be the solutions of equation (1), ordered according to their eigenvalues, $\lambda_{0}<\cdots<\lambda_{-1}$. Thus, the embedding is as follows:

$$
x_{1} \rightarrow y_{1}=A^{T} x_{1} A=\left(a_{1}, a_{1}, \cdots, a_{-1}\right)
$$

where $\mathrm{y}$ is a $l$-dimensional vector, and $\mathrm{A}$ is a $n \times l$ matrix.

Fig. 5.3: Algorithm for Locality Preservation Projection ( Xiaofei He et al, 2014)

\section{Multistage Binary Age Estimation (Msae) Classifiers}

In machine learning, there are a number of classifiers that analyse data for classification and regression. Classifiers like Support Vector Machine (SVM), K-Nearest Neighbour (KNN), and Naïve Bayes etc. are widely used depending on the testing and training dataset. SVM is one such classifier that uses a set of training examples and marks them as belonging to either one of two categories. It is a non-probabilistic binary linear classifier as it builds a model that assigns new examples to either of two categories. SVM builds a model that represents examples as points in space such that separate categories are divided noticeably by a wide gap. Prediction or classification is done on the basis of where the new example is mapped into based on which side of the gap they fall into.

Each stage of the classification procedure decision aiming to determine if the test image of only two non-overlapping age groups. At each stage of the multilayer classification procedure the system uses an independent binary classifier: a single layer feed trained on OLPP features and a support vector machine (SVM). This determines how to narrow down the age groups at the next step based on the probability with which the classification was made and a pre-defined decision table look-up process. The tested age range groups for each stage of classification were also pre-defined. 
Table 3: Accuracy(\%) by Age

\begin{tabular}{lccc}
\hline & $\mathbf{1 8 - 2 9}$ & $\mathbf{3 0 - 4 9}$ & AVERAGE \\
\hline MALE & 65 & 59 & 62 \\
FEMALE & 65 & 62 & 63.5 \\
AVERAGE & 65 & 61 & 63 \\
\hline
\end{tabular}

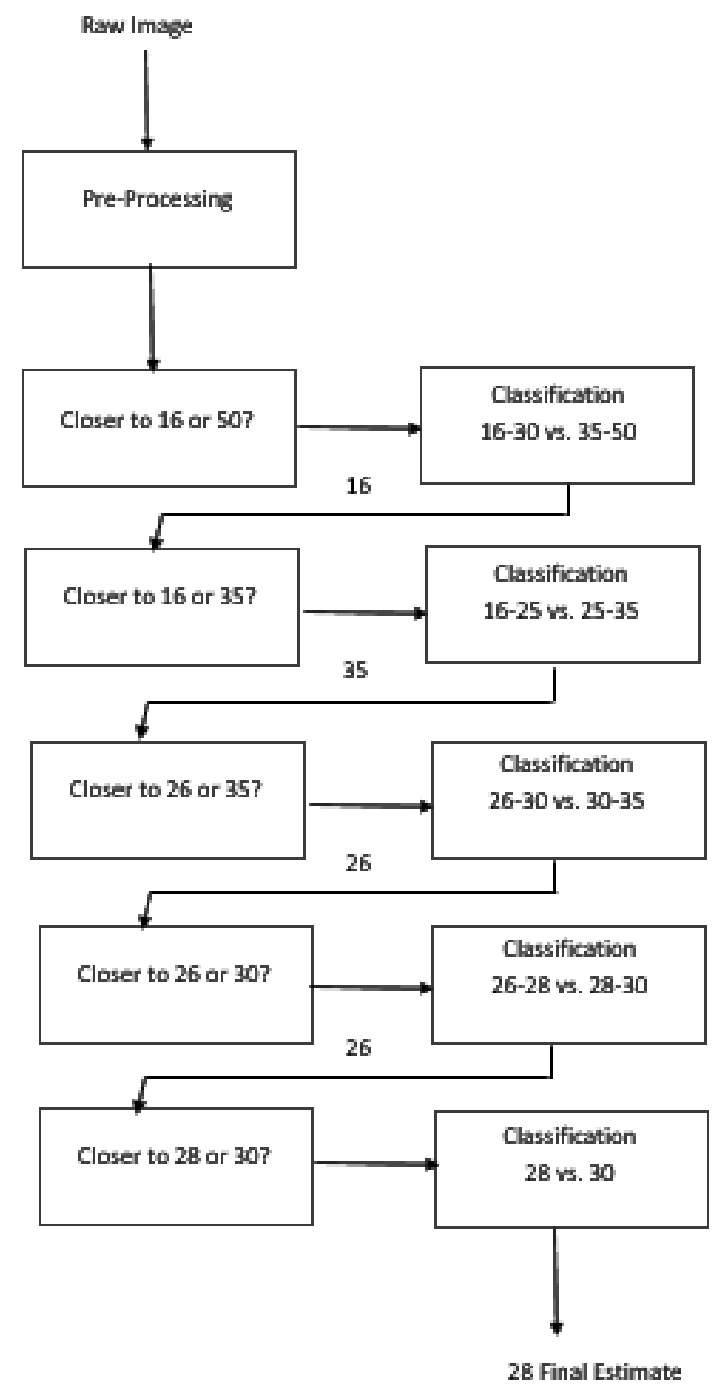

Fig. 5.4: Example for multi stage binary classification (Louis Quinn et al,, 2015)

\section{RESULTS}

The estimated age with a mean error of 5 years provided an average accuracy of $60 \%$ for ages between 18 and 50, and above $65 \%$ for male age groups (18-29) and 65\% for female ages (18-29) irrespective of ethnicity. Average accuracy for ages between 30 and 49 was lower compared to that of 18 and 50 with $61 \%$. Race estimation was prepared using a manual threshold for the Park Aging Mind Laboratory dataset into two categories: African-American and Caucasian. It provided an average accuracy of $98 \%$.

\section{CONCLUSION}

The proposed method used an automatic multi-stage age estimation system (MSAE), designed as support vector machine (SVM) units. The decision making process was based on the orthogonal locality preserved projection (OLPP) and the Sobel edge detector (SED) features extracted from facial images.

The method used was tested using a database containing facial images of participants aged between 18 and 50 and divided into two ethnic groups: African American and Caucasian. The results for male faces showed an average accuracy of $60 \%$ for ages between 18 and 50 , and above $65 \%$ for male age groups (18-29) and $65 \%$ for female ages (18-29) irrespective of ethnicity. Similar trends were observed for all faces however due to smaller number of available valid images of African-American faces, the average accuracy of African-American age estimation was slightly lower than the accuracy achieved for former faces.

The algorithm was trained and tested with a small dataset of $\sim 300$ images in total. Further work could include a larger dataset to improve the accuracy of the algorithm. Also it could include more ethnic groups, increased optimization of the algorithm, or a wider range of ages to estimate age more widely for all age groups. 


\section{REFERENCES}

1. R. Singh, M. Vatsa, A. Noore, and S. K. Singh, "Age Transformation for Improving Face," pp. 576-583, 2007.

2. A. K. Jain, B. Klare, and U. Park, "Face matching and retrieval in forensics applications," IEEE Multimed., vol. 19, no. 1, pp. 20-27, 2012.

3. A. K. Jain, B. Klare, and U. Park, "Face recognition: Some challenges in forensics," 2011 IEEE Int. Conf. Autom. Face Gesture Recognit. Work. FG 2011, pp. 726-733, 2011.

4. H. Han, C. Otto, and A. K. Jain, "Age Estimation from Face Images: Human vs. Machine Performance," vol. 37, no. 6, 2015

5. H. Ling, S. Soatto, N. Ramanathan, and D. W. Jacobs, "A study of face recognition as people age," Proc. IEEE Int. Conf. Comput. Vis., 2007.

6. Z. Li, D. Gong, X. Li, and D. Tao, "Aging Face Recognition: A Hierarchical Learning Model Based on Local Patterns Selection," IEEE Trans. Image Process., vol. 25, no. 5, pp. 2146-2154, 2016.

7. H. Yang, D. Huang, Y. Wang, H. Wang, and Y. Tang, "Face Aging Effect Simulation Using Hidden Factor Analysis Joint Sparse Representation," IEEE Trans. Image Process., vol. 25, no. 6, pp. 2493-2507, 2016.

8. J. Qiu, Y. Dai, Y. Zhang, and J. M. Alvarez, "Hierarchical Aggregation Based Deep Aging Feature for Age Prediction," 2015 Int. Conf. Digit. Image Comput. Tech. Appl. DICTA 2015, 2016.

9. B. Esme and B. Sankur, "Effects of Aging over Facial Feature Analysis and Face Recognition 2 Age Dilemma/ : An Obstacle
, or New," no. March, pp. 1-4, 2010.

10. H. Han, C. Otto, and A. K. Jain, "Age Estimation from Face Images: Human vs. Machine Performance," vol. 37, no. 6, 2015.

11. F. Juefei-xu, K. Luu, M. Savvides, T. D. Bui, and C. Y. Suen, "Investigating Age Invariant Face Recognition Based on Periocular Biometrics," 2011.

12. B. F. Klare, S. Member, and Z. Li, "Matching Forensic Sketches to Mug Shot Photos," vol. 33, no. 3, pp. 639-646, 2011.

13. S. Wang, D. Tao, and J. Yang, "Relative Attribute SVM + Learning for Age Estimation," pp. 1-13, 2015.

14. Xiaofei He ,Partha Niyogi ,2014 August," Locality Preserving Projections" ,IEEE Transaction On Image Processing

15. W. Yu, X. Teng, and C. Liu, "Face recognition using discriminant locality preserving projections," Image Vis. Comput., vol. 24, no. 3, pp. 239-248, 2006.

16. Minear, M. \& Park, D.C. (2004). A lifespan database of adult facial stimuli. Behaviour Research Methods, Instruments, \& Computers. 36, 630-633

7. In Wikipedia from https://en.m.wikipedia.org/ wiki/Sobel operator

18. O. R. Vincent, O. Folorunso, 2009, "A Descriptive Algorithm for Sobel Image Edge Detection", Conference Paper: Proceedings of Informing Science \& IT Education Conference

19. Louis Quinn, Margaret Lech, December 2015, "Multi-Stage Classification Network For Automatic Age Estimation from Facial Images", International Conference on Signal Processing and Communication Systems. 